\title{
DISTRIBUTED UAV-SWARM-BASED REAL-TIME GEOMATIC DATA COLLECTION UNDER DYNAMICALLY CHANGING RESOLUTION REQUIREMENTS
}

\author{
Miguel Almeida ${ }^{\mathrm{a}}$, Hanno Hildmann ${ }^{\mathrm{b}}$ and Gürkan Solmaz ${ }^{\mathrm{c}}$ \\ ${ }^{a}$ NEC Laboratories Europe, Kurfürsten-Anlage 36, D-69115 Heidelberg, Germany, miguel.almeida@neclab.eu

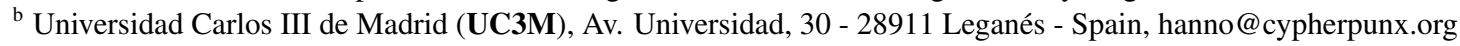 \\ ${ }^{\mathrm{c}}$ NEC Laboratories Europe, Kurfürsten-Anlage 36, D-69115 Heidelberg, Germany, gurkan.solmaz@ neclab.eu
}

KEY WORDS: Self-organization, adaptive behaviour, swarm algorithms, distributed sensing, autonomous decision making

\begin{abstract}
:
Unmanned Aerial Vehicles (UAVs) have been used for reconnaissance and surveillance missions as far back as the Vietnam War, but with the recent rapid increase in autonomy, precision and performance capabilities - and due to the massive reduction in cost and size - UAVs have become pervasive products, available and affordable for the general public. The use cases for UAVs are in the areas of disaster recovery, environmental mapping \& protection and increasingly also as extended eyes and ears of civil security forces such as fire-fighters and emergency response units. In this paper we present a swarm algorithm that enables a fleet of autonomous UAVs to collectively perform sensing tasks related to environmental and rescue operations and to dynamically adapt to e.g. changing resolution requirements. We discuss the hardware used to build our own drones and the settings under which we validate the proposed approach.
\end{abstract}

\section{INTRODUCTION}

Autonomously operating Unmanned Aerial Vehicles (UAVs) have become a major technology in the past decade (though the U.S. military has been using UAVs for operations as far back as the Vietnam War (Broad, 1981)). Due to their low cost and high availability, airborne devices have received much interest from the private consumer, the research community, the industry and the military alike. So-called drones are referred to in the literature as UAVs (Schneider, 2014), UASs (Unmanned Aerial Systems) (Coopmans, 2014), RPAs (Remotely Piloted Aircrafts) (Marcus, 2014) or ROAs (Remotely Operated Aircrafts) (Ogan, 2014).

There are many ways to classify UAVs (Malone et al., 2013). Drone types and capabilities are probably as numerous as the variations on the missions where drones are involved. Some of the larger (fixed wing) UAVs can operate for hours circling over certain areas, e.g., areas contaminated by highly hazardous materials (areas where a manned mission is too dangerous to human life or where providing adequate security for the human operator is simply too costly) (Malone et al., 2013), while the typical flight time of a commercial and publicly available (and affordable) offthe-shelf quadrotor is about 15 to 20 minutes (Erdelj et al., 2017). State of the art UAVs can remain airborne for prolonged periods of time (two weeks (Garber, 2014) or longer) and performance values improve significantly every year (Pauner et al., 2015).

While a lot of research was undertaken regarding the autonomous landing of UAVs in general, few studies have been directed at marine environments where the challenges are more complex or circumstances are more dramatic compared to land or indoor environments. Recently, quadrotor UAVs have been landed with reasonable accuracy on swimming objects and under outdoor conditions (Mendona et al., 2016). The abilities to perform complex maneuvers and to operate as large collectives of units, especially for efficient situational awareness (Erdelj et al., 2017), are going to continue to increase rapidly over the next years.

\subsection{Relevant Application Areas}

The United States Office of the Secretary of Defense (OSD) identifies over twenty UAV mission types, ranging from intelligence, surveillance (Giyenko and Cho, 2016a), reconnaissance missions (Broad, 1981), force protection, firefighting, electronic warfare to communication nodes and others (Malone et al., 2013).

1.1.1 Civil use mission UAVs have recently been used for civil supply, inspection and various search and rescue operations (Cummings et al., 2014). For example, UAVs are used for water management and biofuel production (Coopmans, 2014) or to monitor areas for wild-life protection (Schneider, 2014). For example, the World Wildlife Fund (WWF) controls poaching and illegal wildlife trade (Goodyer, 2013) and in some of Africa's national parks engages perpetrators of such offences (Goodyer, 2013) (though the South African Civil Aviation Authority has banned the use of UAVs in their parks (Andrews, 2014)).

1.1.2 Disaster response and relief applications UAVs can be deployed (Apvrille et al., 2014) during or after disasters to organize disaster management operations, assist the population, reduce the number of victims and mitigate the economic consequences (Tanzi et al., 2014). Disaster scenarios are highly dynamic and authorities normally operate under imperfect information, which directly implies the importance of communication links (Tanzi et al., 2014), as well as the need for real-time changes in surveillance and data collection operations.

UAVs can play a crucial role when existing infrastructure is compromised, malfunctioning or disconnected (Apvrille et al., 2014) or when the environment is deemed too dangerous for humans to operate in (Montufar et al., 2014) by delivering equipment, serving for geographical mapping or vehicular tracking (Giray, 2013). Significant resources have been allocated to develop supervisory control algorithms to assist a single operator and facilitate the remote controlling of multiple UAVs (Cummings et al., 2014). 


\section{SWARM BASED REAL-TIME DATA COLLECTION}

We address the scenarios where a number of UAVs are operating as a single functional unit (a swarm) to provide real-time data from their individual directed sensing equipment (such as onboard cameras). In this context, individual devices provide partial coverage which, when combined with the data from the other devices, offers complete coverage of a target object or area.

The sensing capabilities of any equipment are bounded. Increasing the level of detail (e.g., the resolution of a camera) means reducing the area that is covered. If continuous coverage over an entire area is a hard constraint (as it is for certain search and rescue missions), then this can be achieved by handing over coverage over locations to other devices which currently operate under lower resolution requirements. However, our swarm delivers at least the required resolution, and "operating under lower resolution requirements" does not mean currently delivering only the required minimum resolution. Due to this, handing over locations may affect the performance of the receiving device, possibly resulting in further handovers, in turn causing - in the worst case a ripple effect propagating through the entire swarm.

\subsection{Problem statement}

The fundamental building blocks of the problem are the participating devices $\mathcal{D}$ (the set of drones: $\mathcal{D}=\left\{d_{1}, \ldots, d_{n}\right\}$ ) and $\mathcal{L}$ (all the locations $\mathcal{L}=\left\{l_{1}, \ldots, l_{m}\right\}$ which need to be covered).

Each location $l_{i}$ is defined by its $\mathrm{x}$ - and $\mathrm{y}$-coordinates $\left(\mathrm{x}_{l_{i}}^{\min }, \mathrm{x}_{l_{i}}^{\max }, \mathrm{y}_{l_{i}}^{\min }, \mathrm{y}_{l_{i}}^{\max }\right)$ and a resolution requirement $r e s_{l_{i}}$.

We discretize the locations to have a width and breadth of 1 measurement unit and simplify $l_{i}=\left(\mathrm{x}_{l_{i}}^{\min }, \mathrm{y}_{l_{i}}^{\min }\right.$, res $\left._{l_{i}}\right)$ since $\mathrm{x}_{l_{i}}^{\max }=\left(\mathrm{x}_{l_{i}}^{\min }+1\right)$ and $\mathrm{y}_{l_{i}}^{\max }=\left(\mathrm{y}_{l_{i}}^{\min }+1\right)$.

A drone $d_{j}=\left(x_{d_{j}}, y_{d_{j}}, a_{d_{j}}, \max _{-}\right.$alt $t_{j}$, min_alt $_{d_{j}}$, res $\left._{d_{j}}\right)$ has a position $\left(x_{d_{j}}, y_{d_{j}}\right)$, altitude $a_{d_{j}}$, maximum / minimum altitude (max_alt $t_{d_{j}}$, min_alt $t_{j}$ ) and a sensor resolution res $d_{d_{i}}$.

For a specific solution we distinguish $\mathcal{L}_{a}$, the set of all locations seen at altitude $a$ (with $\mathcal{L}_{a} \subseteq \mathcal{L}$ ) as well as $\mathcal{L}_{d_{i}}$, the set of locations seen by drone $d_{i}$ (with $\mathcal{L}_{d_{i}}=\mathcal{L}_{a_{d_{i}}}$ ); and finally $\mathcal{L}_{d_{i}}^{*}$, the set of locations actually allocated to drone $d_{i}$, (with $\mathcal{L}_{d_{i}}^{*} \subseteq \mathcal{L}_{d_{i}}$ ).

2.1.1 Simplifications Above we have mentioned $\mathrm{res}_{d_{i}}$, the resolution of drone $d_{i}$ 's sensor. Of course the resolution will depend on both the actual resolution of the sensor as well as, in case of a camera, the focal length (i.e., the resolution of the camera as well as its zoom level). In this problem we ignore any zooming capability of the cameras and pretend that this value is fixed.

We treat the surface as a flat area, i.e., we assume that the altitude of a drone correlates to the resolution provided for all locations covered by that UAV, i.e., we do not consider uneven surfaces where, e.g., locations on a hill are covered with a higher resolution due to being closer to the sensor. We further simplify the definition of the covered area by assuming that for each increase in altitude the area of coverage is extended by two location, both in the width as well as in the depth of the covered area. Finally we dictate that there is a maximum altitude max $_{-} a l t_{d_{i}}$ a drone $d_{i}$ can reach and still provide coverage, if this is the same for all drones we use max_alt. The minimum, min_alt, is $=0$.
2.1.2 Coverage and Resolution $\mathcal{L}_{d_{i}}$, the set of locations seen by drone $d_{i}$ depends on $d_{i}$ 's altitude $a_{d_{i}}$ and $\mathrm{x}$ - and y-coordinates $\left(x_{d_{i}}, y_{d_{i}}\right)$ is defined as $\forall j, k \in\left\{1, \ldots, a_{d_{i}}\right\}$ : $\left(x_{d_{i}}, y_{d_{i}}\right),\left(x_{d_{i}}-j, y_{d_{i}}\right),\left(x_{d_{i}}, y_{d_{i}}-k\right),\left(x_{d_{i}}-j, y_{d_{i}}-k\right) \in \mathcal{L}_{d_{i}}$.

The number of locations seen from an altitude $a$ is given by:

$$
\left|\mathcal{L}_{a}\right|=(a \times 2)^{2}
$$

Therefore, $\left|\mathcal{L}_{d_{i}}\right|$, the number of locations that can potentially be covered by a drone $d_{i}$, is determined by the UAV's altitude $a_{d_{i}}$ :

$$
\left|\mathcal{L}_{d_{i}}\right|=\left|\mathcal{L}_{a_{d_{i}}}\right|=\left(a_{d_{i}} \times 2\right)^{2}
$$

The resolution $r_{d_{i}}$ provided by drone $d_{i}$ changes with the altitude and the intrinsic camera values. Normally we would express the resolution of an area in pixels per area and account for parameters like the focal length (zoom level) and would calculate a measure where the higher the value the better the performance. Our actual implementation includes camera resolution, a fixed focal length and the drone's altitude, but here we provide a simplified mathematical model considering only the camera's resolution.

$$
r_{d_{i}}=\frac{\left|\mathcal{L}_{d_{i}}\right|}{\operatorname{res}_{d_{i}}}
$$

We express the current resolution in terms of how much area is contained in a pixel. Minimizing this value across the entire area means to improve the number of pixels per square distance unit.

\subsection{Solution and measure of success}

A solution is instances of $\mathcal{D}$ and $\mathcal{L}$ such that all locations are covered and all resolution requirements are met.

2.2.1 Drone resolution To compare the quality of solutions we define an objective performance measure for the aggregated resolution provided for $\mathcal{L}_{d_{i}}^{*}$ (the set of locations actually allocated to drone $d_{i}$ ) by a drone $d_{i}$ as agg_res $d_{i}$, calculated:

$$
\text { agg_res }_{d_{i}}=r_{d_{i}} \times\left|\mathcal{L}_{d_{i}}^{*}\right|
$$

2.2.2 Swarm resolution The resolution of swarm $\mathcal{D}^{*}$ (i.e., the swarm $\mathcal{D}$ under allocation $\mathcal{L}_{d_{i}}^{*} \subseteq \mathcal{L}_{d_{i}}$ for all its drones $d_{i}$ ) is:

$$
\text { resolution }_{\mathcal{D}^{*}}=\sum_{d_{i} \in \mathcal{D}^{*}} \text { agg_res }_{d_{i}}
$$

Obviously, the lower resolution $\mathcal{D}^{*}$, the better the performance.

2.2.3 Resolution requirements We want to ensure that the resolution requirements are met, i.e., that for all locations $l_{k}$ allocated to a drone $d_{i}$ the resolution $r_{d_{i}}$ provided by that drone $d_{i}$ is equal or higher than $r e s_{l_{i}}: \forall l_{k}: l_{k} \in \mathcal{L}_{d_{i}}^{*} \rightarrow r_{d_{i}} \geq \operatorname{res}_{l_{k}}$.

2.2.4 Performance penalty If allocation $\mathcal{L}_{d_{i}}^{*}$ violates any resolution requirements we define a performance penalty.

The maximum resolution requirement for drone $i$ is:

$$
\max \_r e s\left(\mathcal{L}_{d_{i}}^{*}\right)=\max _{l_{j} \in \mathcal{L}_{d_{i}}^{*}}\left(\operatorname{res}_{l_{j}}\right)
$$

If max_res $\left(\mathcal{L}_{d_{i}}^{*}\right)$ can be used to calculate the maximum altitude max_alt $\left(\mathcal{L}_{d_{i}}^{*}\right)$ then the set of locations covered at this altitude is $\mathcal{L}_{\text {max_alt }\left(\mathcal{L}_{d_{i}}^{*}\right)}$. The set of locations which can not be covered at this altitude $\left(\mathcal{L}_{\text {max_alt }\left(\mathcal{L}_{d_{i}}^{*}\right)}^{-}\right)$is then:

$$
\mathcal{L}_{\text {max_alt }\left(\mathcal{L}_{d_{i}}^{*}\right)}^{-}=\mathcal{L}_{d_{i}}^{*} \backslash \mathcal{L}_{\text {max_alt }\left(\mathcal{L}_{d_{i}}^{*}\right)}
$$


2.2.5 Drone penalty We define penalty $_{d_{i}}$, the number of incremental altitude changes required for drone $d_{i}$ to meet the resolution requirements $\mathcal{L}_{d_{i}}^{*}$ for all its the locations as follows:

$$
\text { penalty }_{d_{i}}=\mathcal{L}_{\text {max_alt }\left(\mathcal{L}_{d_{i}}^{*}\right)}^{-} \times\left(k \times r_{d_{i}}\right)
$$

with $k$ a constant used as a tuning parameter to adjust the impact of the penalty value on the behaviour of the swarm.

The rationale behind our penalty value is that we would like to hand over all locations in $\mathcal{L}_{\text {max_alt }\left(\mathcal{L}_{d_{i}}^{*}\right)}^{-}$to some other UAV. As the size of this st decreases, so should the penalty.

2.2.6 Swarm penalty The penalty penalty $\mathcal{D}^{*}$ of swarm $\mathcal{D}^{*}$ can be calculated as the sum of the penalties of its members:

$$
\text { penalty }_{\mathcal{D}^{*}}=\sum_{d_{i} \in \mathcal{D}^{*}} \text { penalty }_{d_{i}}
$$

2.2.7 Performance evaluation We calculate the performance performance $_{\mathcal{D}^{*}}$ of a swarm $\mathcal{D}^{*}$ using equations 5 and 6 :

$$
\text { performance }_{\mathcal{D}^{*}}=\text { resolution }_{\mathcal{D}^{*}}+\text { penalty }_{\mathcal{D}^{*}}
$$

\subsection{Stochastic re-allocation of locations}

The decision whether or not to re-allocate a location $l_{k}$ from drone $d_{i}$ (the current owner) to $d_{j}$ (a potential new owner) is stochastic, with the probability of a re-allocation being calculated using an evaluation of the current state as well as the potential state (i.e., the state after a re-allocation).

2.3.1 Re-allocation For a re-allocation $\Delta$ of locations from one drone $\left(d_{i}\right)$ to another $\left(d_{j}\right)$ we say that $\mathcal{L}_{d_{i}}^{* \Delta}$ and $\mathcal{L}_{d_{j}}^{* \Delta}$ denote the current allocation of locations and $\mathcal{L}_{d_{i}}^{* \Delta \prime}$ and $\mathcal{L}_{d_{j}}^{* \Delta \prime}$ denote the new allocation, resulting from performing re-allocation $\Delta$.

Exchanges of locations happen always between exactly two drones, $d_{i}$ and $d_{j}$ and on the basis of an estimate of the overall situation before and after a (potential) exchange of locations. Such an estimate will consider the resolution provided by both drones together as well as a penalty for all locations for which the required resolution is not met.

We require that $\left(\mathcal{L}_{d_{i}}^{* \Delta} \cup \mathcal{L}_{d_{j}}^{* \Delta}\right)=\left(\mathcal{L}_{d_{i}}^{* \Delta} \cup \mathcal{L}_{d_{j}}^{* \Delta \prime}\right)$ holds, i.e., the set of locations covered by both drones together does not change.

2.3.2 Optimizing resolution From above we have means to calculate agg_res $\left\{d_{i}, d_{j}\right\}$ for any two drones $i, j$ with $\mathcal{L}_{d_{i}}^{*}$ and $\mathcal{L}_{d_{j}}^{*}$, respectively. We define resolution_before $(\Delta)$ and resolution_after $(\Delta)$ based on $\mathcal{L}_{d_{i}}^{* \Delta}, \mathcal{L}_{d_{j}}^{* \Delta}$ and $\mathcal{L}_{d_{i}}^{* \Delta^{\prime}}, \mathcal{L}_{d_{j}}^{* \Delta \prime}$, respectively:

$$
\begin{gathered}
\text { resolution_before }(\Delta)=\text { agg_res }\left\{d_{i}, d_{j}\right\} \\
\text { resolution_after }(\Delta)=\text { agg_res }\left\{d_{i}, d_{j}\right\}
\end{gathered}
$$

2.3.3 Penalty As above, we define penalty_before $(\Delta)$ and penalty_after $(\Delta)$ based on $\mathcal{L}_{d_{i}}^{* \Delta}, \mathcal{L}_{d_{j}}^{* \Delta}$ and $\mathcal{L}_{d_{i}}^{* \Delta^{\prime}}, \mathcal{L}_{d_{j}}^{* \Delta^{\prime}}$ :

$$
\begin{aligned}
& \text { penalty_before }(\Delta)=\text { penalty }_{\left\{d_{i}, d_{j}\right\}} \\
& \operatorname{penalty\_ after}_{-}(\Delta)=\text { penalty }_{\left\{d_{i}, d_{j}\right\}}
\end{aligned}
$$

2.3.4 Stochastic decision The probability $P_{\Delta}$ of performing re-allocation $\Delta$ is calculated as follows:

$$
P_{\Delta}=\frac{\text { before }(\Delta)^{\alpha}}{\left(\text { before }(\Delta)^{\alpha}+\operatorname{after}(\Delta)^{\alpha}\right)}
$$

with a tuning parameter $\alpha$ and

$$
\begin{gathered}
\operatorname{before}(\Delta)=(\text { resolution_before }(\Delta)+\text { penalty_before }(\Delta)) \\
\operatorname{after}(\Delta)=(\text { resolution_after }(\Delta)+\text { penalty_after }(\Delta))
\end{gathered}
$$

\section{HARDWARE AND SIMULATION FRAMEWORK}

We built and programmed our own UAVs to enable a realistic testing of the proposed approach and to be able to easily showcase the use of a swarm of drones. We furthermore developed a demonstration platform to showcase multi-device solutions / collaboration between devices (e.g., swarms).

\subsection{Drone prototype design}

Our drones are quadcopters with a maximum power demand of $500 \mathrm{~W}$ (the demand when hovering is approx. 70W), on board battery $(11,1 \mathrm{v} 3000 \mathrm{mAh})$, approximate weight of $600 \mathrm{~g}$ (giving us an additional load capacity of around $300 \mathrm{~g}$ ). The projected flight time for use in demos (outside and subjected to environmental conditions but without additional load) is 15 minutes. The dimensions are $177 \mathrm{~mm} \times 177 \mathrm{~mm} \times 192 \mathrm{~mm}$ (cf. Fig. 1).

3.1.1 Control module (CM) As not uncommon in the literature (Mhatre et al., 2015) (Choi et al., 2016) (Giyenko and Cho, 2016b), the onboard computing platform (running the control module (CM), the simulated mobile platform as well as the optional simulated sensor platform is a Raspberry Pi 2 (see Fig. 1) with the following specifications: a $900 \mathrm{MHz}$ quad-core ARM Cortex-A7 CPU, 1GB RAM, 4 USB ports, 40 GPIO pins, Full HDMI port, Ethernet port, combined $3.5 \mathrm{~mm}$ audio jack and composite video, camera interface (CSI), display interface (DSI), Micro SD card slot, VideoCore IV 3D graphics core. It is running Linux (Raspbian) as operating system and ROS (BSD or LGPLv3 or GPLv3 License) over VPN to connect to other modules.

We used MAVROS to publish the autopilot's / SITL's mavlink data to the network, relays it to GCS (Ground Control Software) and furthermore relays the CM's instructions to the autopilot. The Ardupilot SITL (SW in the loop, GPLv3 License, cf., e.g,. (Bupe et al., 2015) (Rojas et al., 2015) (de Albuquerque et al., 2016) (Guevara et al., 2015) (Mukherjee et al., 2014)) autopilot software facilitates the simulation of flight operations and enables us to use additional Raspberry Pis to simulate larger swarms.

3.1.2 Flight module (FM) The flight module (the hardware realizing all the flight operations, also used in, e.g., (Bupe et al., 2015) (Choi et al., 2016) (Giyenko and Cho, 2016b)) is a Pixhawk (see Fig. 1) featuring a 168Mhz 32-Bit STM32F427 Cortex M4, 256KB RAM, 2MB Flash, 14 PWM / servo outputs as well as connectivity options for additional peripherals (UART, I2C, CAN) and redundant power supply inputs as well as an automatic failover. Regarding peripheral sensors we are currently using only GPS, Airspeed sensor, Sonar, LiDar and Optical Flow, but the Pixhawk is not restricted to these and additional sensors can be added. The pixhawk is licensed under the Creative Commons License (Open-Source HW). We used APM Flight Stack (GPLv3 License). This connects to the navigation sensors (e.g., GPS) and controls all basic flight / navigation dynamics. 

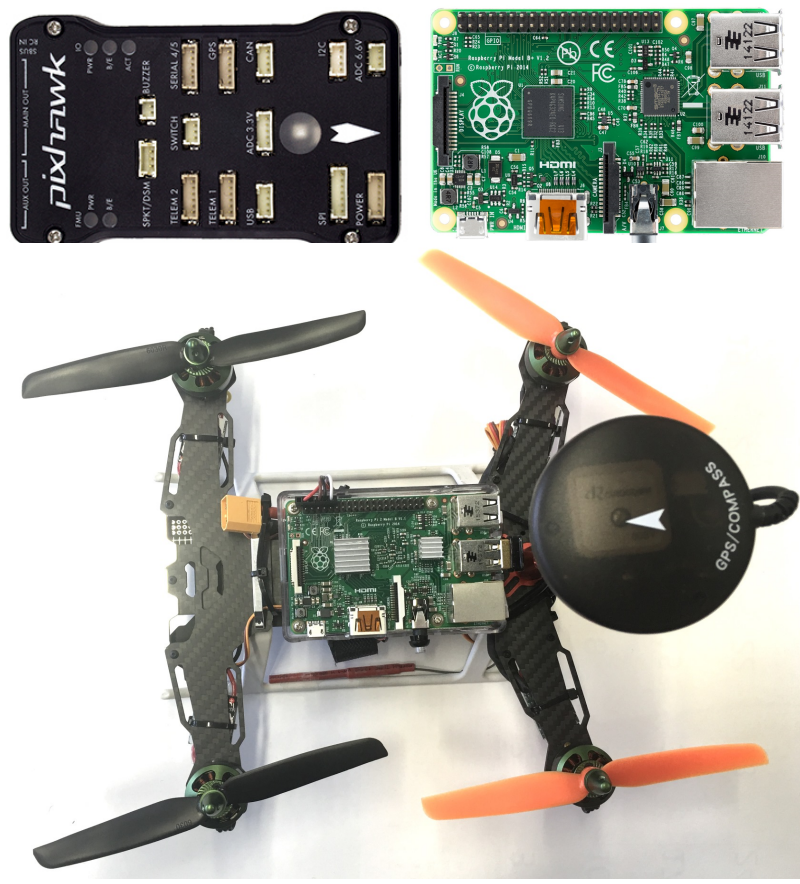

Figure 1. (top left) The pixhawk flight module / autopilot, (top right) the Raspberry Pi 2 and (bottom) one of the NEC MSP prototypes used for the evaluation and testing of the approach.

\subsection{Simulation / Testing Environment}

The process of designing and implementing distributed algorithms for the control of swarms poses the challenge of evaluating and testing them in the real world, that is, using actual hardware and letting it operate in a physical environment. UAV use and availability is spreading faster than awareness of - or legislative frameworks to address - related concerns (Pauner et al., 2015). Their rapid adoption outpaces legal, policy, and social ability to cope with issues regarding privacy and interference with wellestablished commercial air space (Sterbenz, 2016). Regulations differ between countries (Erdelj et al., 2017) and have to address a number of technical and societal concerns and challenges (Vattapparamban et al., 2016). (Altawy and Youssef, 2016) surveys the main security, privacy, and safety aspects associated with the use of civilian drones in the national airspace.

By their nature (distributed and intended for swarms), the proposed algorithms require a substantial number of devices in order to show the intended benefit. The device type itself poses the problem of being subjected to a variety of different legal requirements depending on where (location) and under which circumstances (commercial, non-commercial, etc) they are operated.

The demonstration platform currently under development will address these issues by (a) facilitating the use of large numbers of simulated devices to augment a small swarm of physical devices; and (b) enabling the co-location of physical devices that are factually not in the same place. While the latter makes it possible to share resources between different labs, it is primarily aimed at enabling us to perform demonstrations in areas where the legal restrictions for the operation of physical drones would otherwise prevent the demonstration (or make it exceedingly expensive).

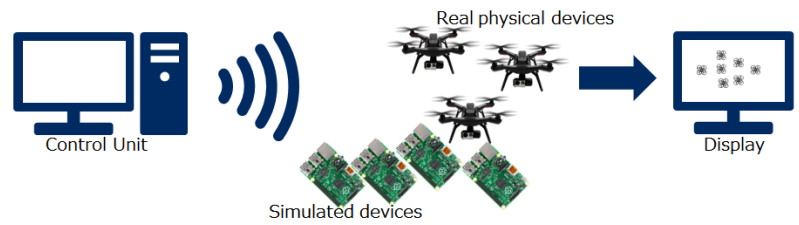

Figure 2. Parts of the demonstration platform: control unit, physical devices, simulated devices and a visualization module.

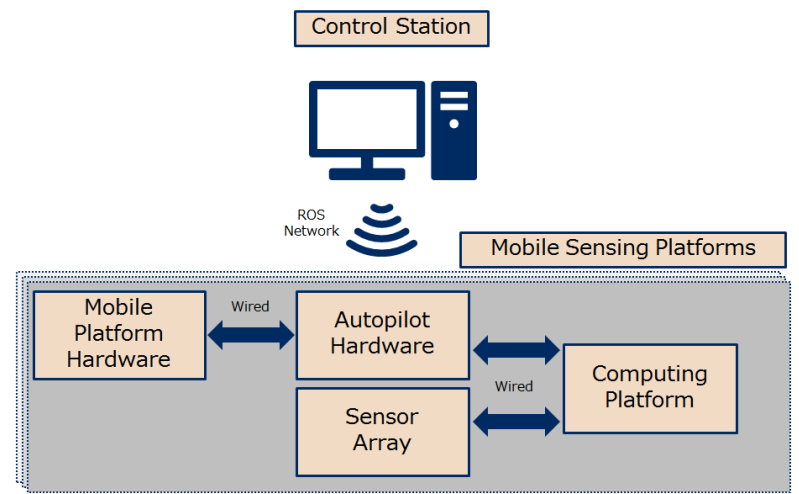

Figure 3. A conceptual overview over the mobile sensing platform (the hardware is shown in Figure 1).

Figure 2 shows the 4 components of the platform: (1) a control interface to run the simulation, (2) physical devices (possibly in a number of different locations), (3) simulated devices and finally (4) a visualization module showing the scenario.

We designed the platform to be able to handle a number of different devices, i.e., it is not restricted to our quadcopters or, for that matter, to drones in general. Specifically the use with fixed-wing drones as well as rovers (unmanned ground vehicles) is possible and simulations could use all of these device types together.

The current project only considers one type of drone, but planning for this functionality already sets the stage for future projects that focus on inter-swarm collaboration and enables us to evaluate the performance of our algorithms in a broader context.

The control unit as well as the visualization tool are realized in a control station (a PC or a laptop), which communicates through a wireless network with the members of the swarm (cf. Fig. 3).

We do not distinguish between virtual and physical devices or even the device type (though in the foreseeable future we will only be using quadcopters) and call all of them mobile sensing platforms (MSPs) as in Fig. 3.

Within each MSP (of which there can be many) we distinguish between (a) the computing platform where the NEC proprietary algorithms are used (this is called command module (CM) and with the exception of the flight module, all elements shown are part of the CM), (b) a sensor array (real or simulated), (c) the flight module (the hardware controlling the drone) and (d) the mobile hardware platform (i.e., the drone).

The demonstration platform uses the ROS network as it provides all functionalities required for staging the demonstrations. 


\subsection{Implementation}

The implementations required code for two physically separate parts of the demonstration platform: the control station (containing the Dispatch module) and UAV. For either algorithm implementation, the Dispatch module was the only module on the control station side for which code needed to be written (for the implementation of the algorithm; there was additional code written for the implementation of the System Control to handle, e.g., UAV registry). On the UAV side, the only module that was concerned was the Computing Platform part of the MSP.

The control station is a laptop (running instances of Dispatch, Environment and System Control) while, as discussed, the Computing Platform on our UAVs was a Raspberry Pi 2.

3.3.1 Control station side (Dispatch module) On the control station side (in our case the laptop used to supervise the operations) only the Dispatch module is involved because this is where all altitude control relevant interaction between UAVs and the control station is happening. That is, any communication between the UAVs and the control station which is related to an ongoing swarm surveillance operation happens exclusively through the Dispatch, be it for general day-to-day operations as well as for dedicated and task specific operations (such as, e.g., "providing sensor readings for location $L$ " or, as in this case, "orchestrating UAV swarm based video surveillance for area A”).

For the altitude control algorithm we assume that the high level task of controlling the swarm is handled by a compartmentalized sub-system. Due to this we implemented a system that allows UAVs to sign on to it as participating members (having been instructed to do so by another module sub-system of Dispatch) and which will be the interface between the application side (whatever it may be) and the UAV swarm.

In a fully developed and deployed system Dispatch would also handle the data streams generated by the UAVs (e.g., video streams, coming from different UAVs and, together, covering all locations in the target area). Dispatch would combine these and pass them through to either the software analyzing the data or to the output device specified by the human operator.

3.3.2 UAV / drone side (MSP control module) For the UAV implementation the choice for either a centralized or a decentralized approach had to be made. Since the algorithm is inherently intended to be decentralized the choice is determined by operational and practical considerations: in the implementation of the simulation and demonstration platform (using ROS as communication framework) we found that the communication overhead was large because coordinating asynchronous communications between many different devices required a lot of time.

We implemented a centralized version of the approach where the code running on the laptop is coordinating the swarm: It performs all the calculations and periodically updates the swarm accordingly. However, this centralized version can also be implemented to run on one of the UAVs which then performs all the calculations and once a decision to re-allocate a location is made this "master drone" simply updates the swarm accordingly.

In the de-centralized approach, all UAVs compete for locations and interactions between UAVs are always between two UAVs that can both cover a specific location. Our decision to implement the algorithm in a centralized form was due to the inherent
TCP/IP and wireless latency. A message round trip will take approx $20 \mathrm{~ms}$ which would mean that demonstrations would work slower than preferred. This is an issue that can be addressed by better or different communication architectures. We don't foresee any major obstacles when implementing the algorithm for a fleet of real UAVs. At the moment, the centralized implementation allows us to evaluate the approach without loss of generality.

3.3.3 Communication protocols The Dispatch module handles all issues related to communication with the swarm. UAVs sign on to a surveillance swarm and Dispatch supplies them with current and updated resolution requirements. The individual UAVs in return supply the Dispatch with a series of video streams (as well as an indication which areas these relate to).

\section{RESULTS AND EVALUATION}

To test the approach we created a swarm of 25 UAVs and used an area of $20 \times 20$ locations. UAVs were positioned at the intersection of 4 locations and distributed homogeneously over the area. Figure 4 shows the set up of the simulated area.

UAV altitudes were discrete: there were 11 altitudes ranging from zero (ground level) to $200 \mathrm{~m}$ (consequently, at 10 of these 11 altitudes coverage over increasingly large areas is provided). UAVs started at 200 meters (the highest altitude where minimum resolution requirements could be met) but could ascend to higher altitudes (and in the initial phase of a simulation some of them do, so as to facilitate the trading of locations with UAVs that are further away) in steps of $32.05371 \mathrm{~m}-200 \mathrm{~m}$. All UAVs initially started at altitude $200 \mathrm{~m}$ and then descended to the initial location.

Whenever not used to control a real UAV, multiple instances of a simulated MSP were executed on a single Raspberry Pi. This implies that the results are, if anything, on the cautious side as we used only a fraction of the available computation power to run the individual UAVs. For an eventual implementation of the system we would use a more powerful computer at the Dispatch side to improve performance even further.

When simulating the surveillance swarm we ignored zooming of cameras altogether. The reason for this is that the algorithm is effectively trading off covered area for quality of video feed, and both, the changes in altitude or the zooming cause the same changes in coverage and quality. Implementing a realistic model for both would have caused a lot of extra work without providing any benefit as far as the evaluation of the algorithm is concerned.

In a trial implementation we would improve the actual delivered quality by making use of both mechanisms: since zooming will most likely be a lot faster and a lot cheaper (energy wise), we will use zooming to cover changes quickly and then adapt the altitude of the UAVs to ensure zooming never leaves the UAV at one end of the spectrum (fully zoomed in or out).

The camera values used in the simulations were taken from a Sony NEX 5R camera with a 25mm lens: pixels $\mathrm{h}=4592$ (number of pixels in the $\mathrm{Y}$ axis of the sensor), pixels_v=3056 (number of pixels in the $X$ axis of the sensor), sensor_h=23.4 (sensor size in the $y$ axis), sensor_v=15.6 (sensor size in the $\mathrm{x}$ axis). As the horizontal resolution was marginally better than the vertical resolution all reported values refer to the vertical to ensure we can deliver at least the reported quality. Along these lines, we simplified the area to be a square (not, as it really is, a rectangle) and ignored the additional area coverage. 


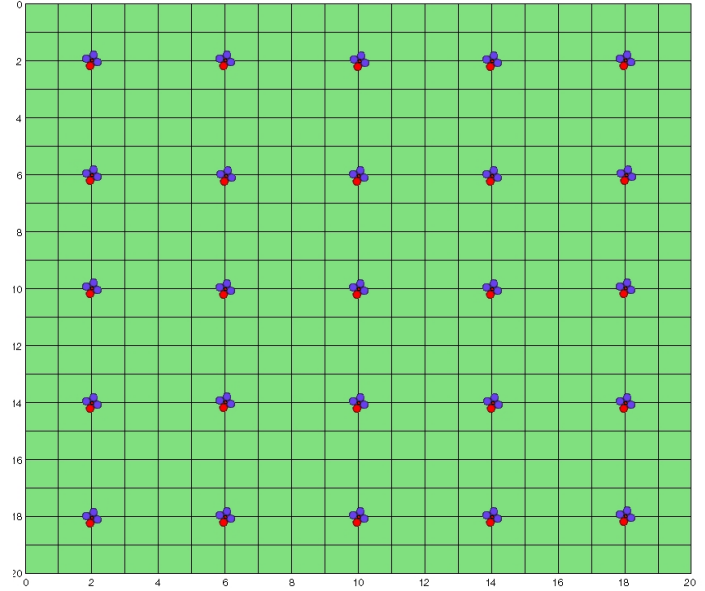

Figure 4 . The test area (400 locations arranged in a $20 \times 20$ grid) and the 25 UAVs in homogeneous swarm formation.

\subsection{Baseline performance}

We first establish a baseline performance for the algorithm by using it to optimize the altitude of a swarm of UAVs in the absence of resolution requirements. Due to the homogenous distribution of the UAVs (cf. Fig. 4) we know that the best possible solution is when all UAVs have descended to the altitude when they can cover two fields in any direction (i.e., an area of $4 \times 4$ locations). Given that the distribution of the UAVs allows for a unique best possible solution, we can test the convergence property of the algorithm. And indeed, the graphs in Fig,. 6 show that the swarm converges quickly towards the best solution. The standard deviation shows that after a brief initial phase the UAVs do not only decrease their altitudes but do so increasingly coherent until they all arrive at, and hold, the same altitude.

This already shows that the approach does indeed perform as intended / predicted. In the following sections we will take a closer look at the performance of the algorithm when resolution requirements are added and removed, i.e., for cases when the optimal solution is not as straight forward to find as in the baseline case.

\subsection{Performance under dynamic requirement changes}

To test the performance of the algorithm when the resolution requirements are not uniformly distributed / the same everywhere we added specific resolution requirements during the simulation. The following resolution requirements were added (cf. Fig. 5):

- until iteration 1999 no requirements are present, all drones descend to their optimal altitude.

- at iteration 2000 two resolution requirements are added for locations 6,6 and 14,14. At $3.27 \mathrm{~mm} /$ pixel this forces the closest UAV to descend to altitude 1 in order to meet the resolution requirements and the previously covered $4 \times 4$ locations have to be handed over to neighbouring drones.

- at iteration $\mathbf{4 0 0 0}$ the previous requirements are moved to locations 6,14 and 14,6 by removing them at iteration 3999 and adding the new ones at iteration 4000 .

- at iteration 6000 existing requirements are removed in the previous iteration and one is added for location 10,10.

- at iteration 8000 the initial two requirements are repeated.

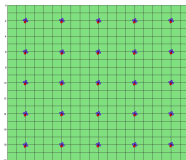

(a)

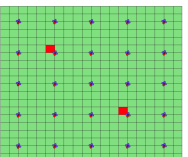

(b)

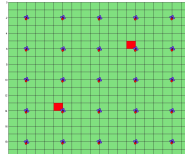

(c)

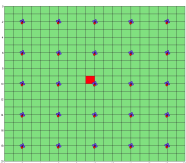

(d)
Figure 5. Requirements: iterations 0-1999, (a) none, (b) 2000-3999, (c) 4000-5999, (d) 6000-7999 and (b) 8000-end.

\subsection{Individual altitudes}

In the baseline scenario all drones converged on the same altitude and into a stable formation (cf. Fig. 6). Under changing resolution requirements the optimal solution(s) are less stable and the occasional space exploration occurs (cf. Fig. 7).

The next two graphs (cf. Figures 8 and 9) show the various altitudes at which drones are found throughout the surveillance simulation. These represent the actual altitudes under the simplification that disallows zooming. For a full deployment we would make use of the camera zoom and then have the drones follow, in other words the actual image quality can be expected to be better (the delivery of increased resolution to be faster and smooth) than the altitude changes plotted in the graphs. To have a closer look at the altitudes we provide the frequency distribution for the swarm's altitudes. We can see that after the initial allocation and optimization, the vast majority of UAVs remains either on altitude 2 (the optimal altitude in the absence of any resolution requirement) and altitude 3 . Only 1 or 2 UAVs are observed at altitudes 4 and 5. These are the UAVs that have to increase their altitude to cover locations previously allocated to a UAV that is now flying closer to a location with resolution requirements.

\subsection{Discussion}

The benchmark scenario shows the drones continuously improving on their initial performance and settling into the (obvious) best possible solution. When increased resolution requirements are imposed, the swarm responds through altitude changes by individual drones, some to provide the required solution and some to take over the areas previously covered by the former. In our evaluation we were mainly interested in the ability of the swarm to consistently converge on good solutions and less on the response time. The motivation for this is that the response time directly responds to the number of iterations the swarm / individual drones can perform per second, which in turn depends on the hardware available. We argue that response times can be improved through (a) more powerful on-board computing hardware or (b) the use of cloud based resources or a dedicated control computing facility. In addition, the actual response time required to change the altitude can be off-set through the use of zooming.

\section{CONCLUSION}

The presented approach enables swarm of devices to collaboratively cover an area and provide continuous data quality for, e.g., video coverage, even if the resolution requirements for individual locations are subject to change. The approach is scalable and the swarm used for the evaluation is already large enough to deliver good results; performance will only increase with larger swarms. Due to legal and practical considerations the discussed evaluation is - in fact - merely a proof of concept case but future projects are aiming at, e.g., disaster response systems, i.e., real world scenarios where normal regulations and norms are often suspended. 


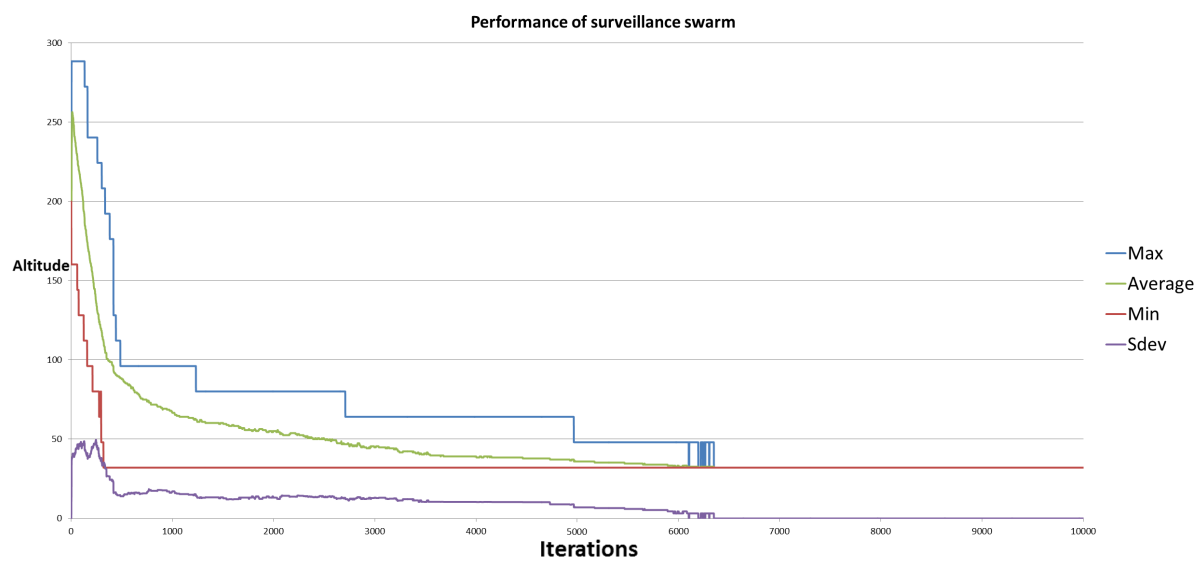

Figure 6. The coverage problem without requirements, baseline performance: homogeneous altitudes (best possible solution).

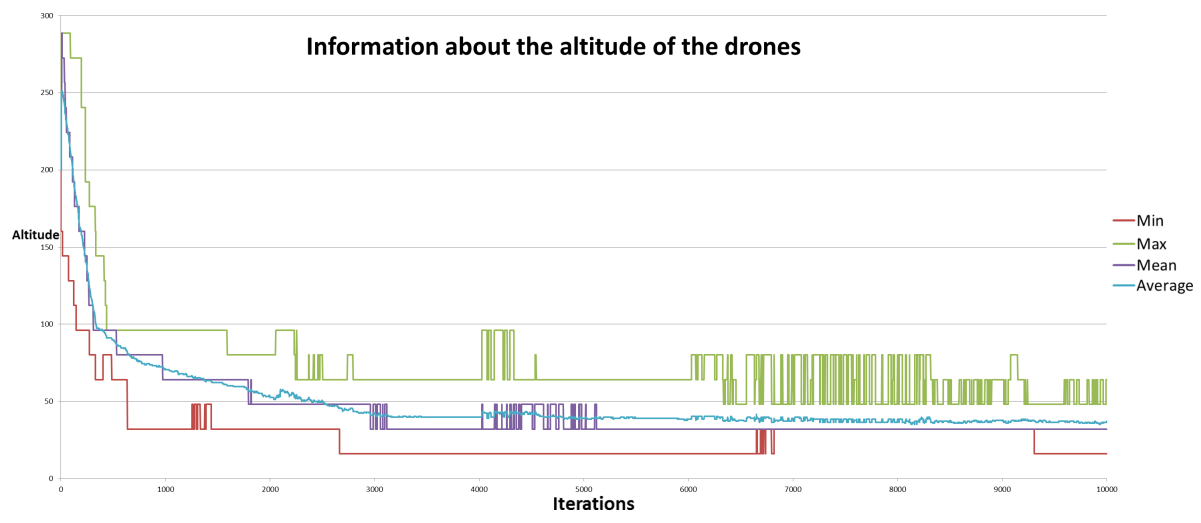

Figure 7. The altitudes of the UAVs in the surveillance swarm under the changing resolution requirements (cf. Fig. 5).

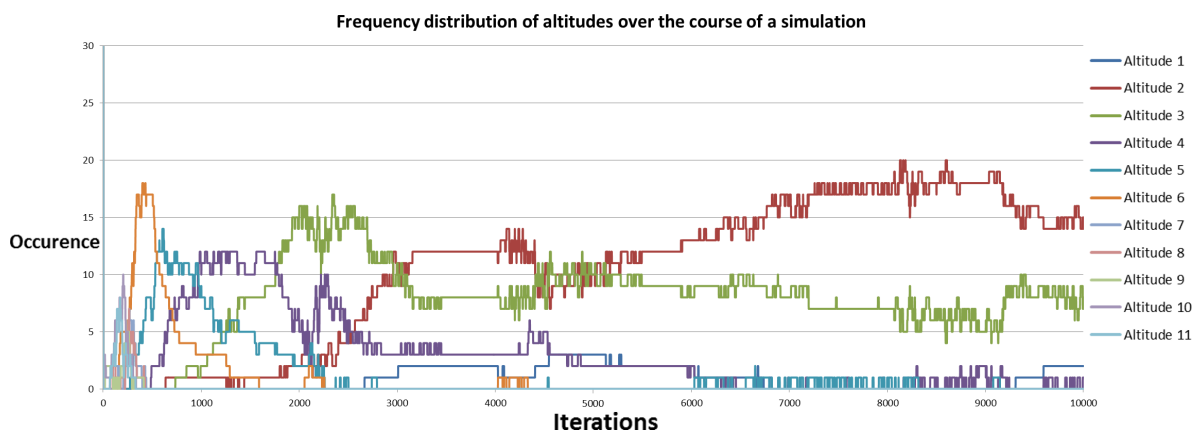

Figure 8 . The frequency distribution for the altitudes of the drones.

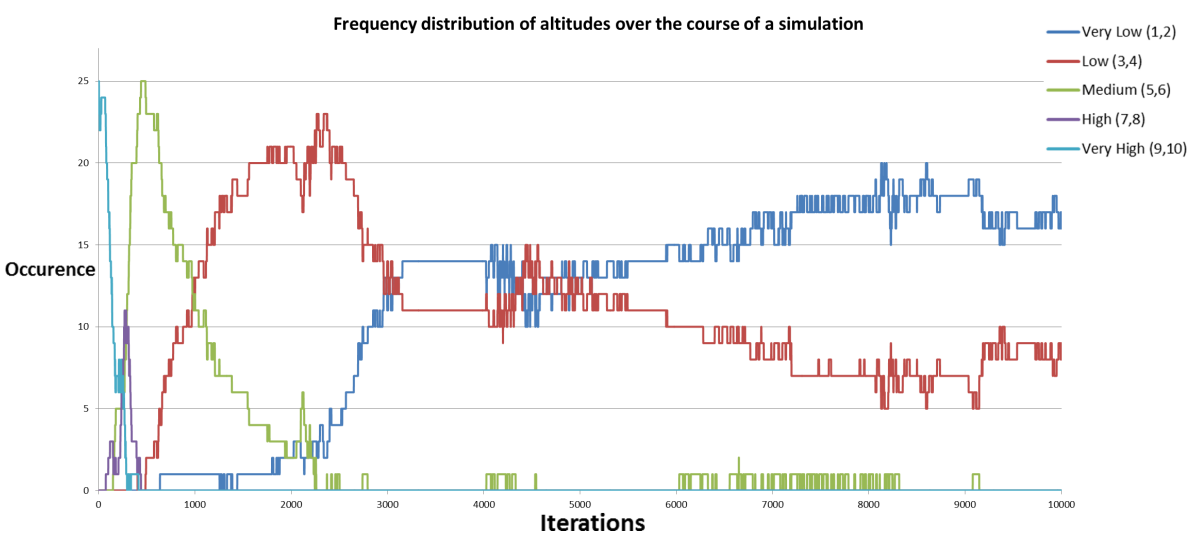

Figure 9. An aggregated version of the drones' altitudes. 


\section{ACKNOWLEDGEMENTS}

The presented work was undertaken as part of a project by NEC Laboratories Europe, the approach is the subject of a pending patent application (PCT/EP 2015/062810 (pending))

\section{REFERENCES}

Altawy, R. and Youssef, A. M., 2016. Security, privacy, and safety aspects of civilian drones: A survey. ACM Trans. CyberPhys. Syst. 1(2), pp. 7:1-7:25.

Andrews, C., 2014. UAVs in the wild. Engineering Technology 9(7), pp. 33-35.

Apvrille, L., Tanzi, T. and Dugelay, J.-L., 2014. Autonomous drones for assisting rescue services within the context of natural disasters. In: General Assembly and Scientific Symposium (URSI GASS), 2014 XXXIth URSI, pp. 1-4.

Broad, W. J., 1981. The U.S. flight from pilotless planes. Science 213(4504), pp. 188-190.

Bupe, P., Haddad, R. and Rios-Gutierrez, F., 2015. Relief and emergency communication network based on an autonomous decentralized UAV clustering network. In: SoutheastCon 2015, pp. 1-8.

Choi, H., Geeves, M., Alsalam, B. and Gonzalez, F., 2016. Open source computer-vision based guidance system for UAVs onboard decision making. In: 2016 IEEE Aerospace Conference, pp. 1-5.

Coopmans, C., 2014. Architecture requirements for ethical, accurate, and resilient unmanned aerial personal remote sensing. In: Unmanned Aircraft Systems (ICUAS), 2014 International Conference on, pp. 1-8.

Cummings, M., Bertucelli, L., Macbeth, J. and Surana, A., 2014. Task versus vehicle-based control paradigms in multiple unmanned vehicle supervision by a single operator. HumanMachine Systems, IEEE Transactions on 44(3), pp. 353-361.

de Albuquerque, J. C., de Lucena, S. C. and Campos, C. A. V., 2016. Evaluating data communications in natural disaster scenarios using opportunistic networks with unmanned aerial vehicles. In: 2016 IEEE 19th International Conference on Intelligent Transportation Systems (ITSC), pp. 1452-1457.

Erdelj, M., Natalizio, E., Chowdhury, K. R. and Akyildiz, I. F., 2017. Help from the sky: Leveraging UAVs for disaster management. IEEE Pervasive Computing 16(1), pp. 24-32.

Garber, L., 2014. News briefs. Computer 47(6), pp. 12-17.

Giray, S., 2013. Anatomy of unmanned aerial vehicle hijacking with signal spoofing. In: Recent Advances in Space Technologies (RAST), 2013 6th International Conference on, pp. 795-800.

Giyenko, A. and Cho, Y. I., 2016a. Intelligent UAV in smart cities using iot. In: 2016 16th International Conference on Control, Automation and Systems (ICCAS), pp. 207-210.

Giyenko, A. and Cho, Y. I., 2016b. Intelligent unmanned aerial vehicle platform for smart cities. In: 2016 Joint 8th International Conference on Soft Computing and Intelligent Systems (SCIS) and 17th International Symposium on Advanced Intelligent Systems (ISIS), pp. 729-733.
Goodyer, J., 2013. Drone rangers [africa special sustainability]. Engineering Technology 8(5), pp. 60-61.

Guevara, K., Rodriguez, M., Gallo, N., Velasco, G., Vasudeva, K. and Guvenc, I., 2015. UAV-based gsm network for public safety communications. In: SoutheastCon 2015, pp. 1-2.

Malone, P., Apgar, H., Stukes, S. and Sterk, S., 2013. Unmanned aerial vehicles unique cost estimating requirements. In: Aerospace Conference, 2013 IEEE, pp. 1-8.

Marcus, M., 2014. Spectrum policy challenges of UAV/drones [spectrum policy and regulatory issues]. Wireless Communications, IEEE 21(5), pp. 8-9.

Mendona, R., Marques, M. M., Marques, F., Loureno, A., Pinto, E., Santana, P., Coito, F., Lobo, V. and Barata, J., 2016. A cooperative multi-robot team for the surveillance of shipwreck survivors at sea. In: OCEANS 2016 MTS/IEEE Monterey, pp. 1-6.

Mhatre, V., Chavan, S., Samuel, A., Patil, A., Chittimilla, A. and Kumar, N., 2015. Embedded video processing and data acquisition for unmanned aerial vehicle. In: 2015 International Conference on Computers, Communications, and Systems (ICCCS), pp. 141-145.

Montufar, D., Munoz, F., Espinoza, E., Garcia, O. and Salazar, S., 2014. Multi-UAV testbed for aerial manipulation applications. In: Unmanned Aircraft Systems (ICUAS), 2014 International Conference on, pp. 830-835.

Mukherjee, A., Chakraborty, S., Azar, A. T., Bhattacharyay, S. K., Chatterjee, B. and Dey, N., 2014. Unmanned aerial system for post disaster identification. In: International Conference on Circuits, Communication, Control and Computing, pp. 247-252.

Ogan, R., 2014. Integration of manned and unmanned aircraft systems into U.S. airspace. In: SOUTHEASTCON 2014, IEEE, pp. 1-4.

Pauner, C., Kamara, I. and Viguri, J., 2015. Drones. current challenges and standardisation solutions in the field of privacy and data protection. In: 2015 ITU Kaleidoscope: Trust in the Information Society (K-2015), pp. 1-7.

Rojas, A. J., Gonzalez, L. F., Motta, N. and Villa, T. F., 2015. Design and flight testing of an integrated solar powered UAV and wsn for remote gas sensing. In: 2015 IEEE Aerospace Conference, pp. 1-10.

Schneider, D., 2014. Open season on drones? Spectrum, IEEE 51(1), pp. 32-33.

Sterbenz, J. P., 2016. Drones in the smart city and iot: Protocols, resilience, benefits, and risks. In: Proceedings of the $2 \mathrm{Nd}$ Workshop on Micro Aerial Vehicle Networks, Systems, and Applications for Civilian Use, DroNet '16, ACM, New York, NY, USA, pp. 3-3.

Tanzi, T., Apvrille, L., Dugelay, J.-L. and Roudier, Y., 2014. UAVs for humanitarian missions: Autonomy and reliability. In: Global Humanitarian Technology Conference (GHTC), 2014 IEEE, pp. 271-278.

Vattapparamban, E., Gven, ., Yurekli, A. ., Akkaya, K. and Uluaa, S., 2016. Drones for smart cities: Issues in cybersecurity, privacy, and public safety. In: 2016 International Wireless Communications and Mobile Computing Conference (IWCMC), pp. 216-221. 